\title{
A Note on the Decrease of Truncated Wightman Functions for Large Space-like Separation of the Arguments
}

\author{
K. Pohlmeyer \\ II. Institut für Theoretische Physik der Universität Hamburg
}

Received November 27, 1967

\begin{abstract}
The truncated Wightman functions cannot decrease arbitrarily fast for large space-like separation of the arguments. For certain configurations they can fall off at most exponentially.
\end{abstract}

Upper bounds on the decrease of truncated Wightman functions were established a long time ago $[1-5]$. For instance, for a relativistic quantum field theory of a self-interacting neutral, scalar field $A(x) \mathrm{H}$. ARAKI [2] (compare the footnote in [5]) proved the following theorem: Under the assumptions of a) Lorentz invariance, b) temperedness of the Wightman functions, c) the existence of a lowest non-zero mass, the truncated vacuum expectation value (TVEV)

$$
\left\langle A\left(x_{0}\right) \ldots A\left(x_{n}\right)\right\rangle^{T}
$$

vanishes at least exponentially for $x_{i-1}-x_{i}=\xi_{i}+\lambda \xi_{i}^{\prime} i=1, \ldots, n$ where $\xi_{i}+\lambda \xi_{i}^{\prime}$ should be a Jost point for sufficiently large $\lambda$ and $\lambda \rightarrow+\infty, \xi_{i}, \xi_{i}^{\prime}$ fixed (with at least one $\xi_{i}^{\prime} \neq 0$ ).

Here we want to point out that a lower bound on the decrease of the TVEV for similar configurations can be obtained as well. We do not assume locality or the existence of a lowest non-zero mass.

To begin with, let us consider the 2-point function. Lorentz invariance, temperedness and positive definiteness imply the well-known Källén-Lehmann representation

$$
\left\langle A\left(x_{0}\right) A\left(x_{1}\right)\right\rangle^{T}=\left\langle A\left(x_{0}\right) A\left(x_{1}\right)\right\rangle=i \int_{0}^{\infty} d \varrho(\mu) \Delta_{\mu}^{+}\left(x_{0}-x_{1}\right),
$$

$\varrho(\mu)$ a positive tempered measure

$$
\sim \frac{\text { const }}{-\left(x_{0}-x_{1}\right)^{2}}
$$

(or $\sim \frac{\sqrt{m}}{2^{5 / 2} \pi^{3 / 2} \sqrt{-\left(x_{0}-x_{1}\right)^{2^{3 / 2}}}} \exp \left\{-m \sqrt{-\left(x_{0}-x_{1}\right)^{2}}\right\}$ in case of the existence of a lowest non-zero mass $m$ in the theory).

Next, we turn to the 3-point function. It is analytic in the "extended tube" $\mathscr{T}_{0,1,2}^{\prime}$ the boundaries of which are explicitly known in terms of the invariants [6]. Consider

$$
W_{2}^{T}\left(x_{0}, x_{1}, x_{2}\right)=\left\langle A\left(x_{0}\right) A\left(x_{1}\right) A\left(x_{2}\right)\right\rangle^{T}
$$


for $x_{i-1}-x_{i}=\xi_{i}+\lambda \xi_{i}^{\prime} i=1,2$ with $x_{0}, x_{1}, x_{2}$ totally space-like in the order $0,1,2$ for sufficiently large positive $\lambda, \xi_{i}, \xi_{i}^{\prime} i=1,2$ fixed, not both $\xi_{i}^{\prime}=0$.

Define

$$
w_{2}^{T}\left(\lambda ; \xi_{i}, \xi_{i}^{\prime}\right)=W_{2}^{T}\left(x_{0}, x_{1}, x_{2}\right) .
$$

$w_{2}^{T}\left(\lambda ; \xi_{i}, \xi_{i}^{\prime}\right)$ is real-analytic for sufficiently large positive $\lambda$ and can be analytically continued in $\lambda$ into a wedge-shaped region with the following angle :

$$
\begin{aligned}
& \pi-\operatorname{arctg} \sqrt{\frac{\xi_{1}^{\prime 2} \xi_{2}^{\prime 2}-\left(\xi_{1}^{\prime} \cdot \xi_{2}^{\prime}\right)^{2}}{\left(\xi_{1}^{\prime} \cdot \xi_{2}^{\prime}\right)^{2}}} \text { if } \xi_{i}^{\prime} \cdot \xi_{j}^{\prime}<0 i, j=1,2, \xi_{1}^{\prime 2} \xi_{2}^{\prime 2}>\left(\xi_{1}^{\prime} \cdot \xi_{2}^{\prime}\right)^{2} \\
& \pi \\
& \operatorname{arctg} \sqrt{\frac{\xi_{1}^{\prime 2} \xi_{2}^{\prime 2}-\left(\xi_{1}^{\prime} \cdot \xi_{2}^{\prime}\right)^{2}}{\left(\xi_{1}^{\prime} \cdot \xi_{2}^{\prime}\right)^{2}}} \quad \text { if } \quad \xi_{1}^{\prime 2}<0, \quad \xi_{2}^{\prime 2}<0, \quad \xi_{1}^{\prime} \cdot \xi_{2}^{\prime} \geqq 0 \\
& \pi \\
& \xi_{1}^{\prime}=0, \xi_{2}^{\prime 2}<0, \xi_{1} \cdot \xi_{2}^{\prime}<0 \\
& \text { if } \text { or } \xi_{1}^{\prime 2}<0, \xi_{2}^{\prime}=0, \xi_{1}^{\prime} \cdot \xi_{2}<0 \\
& 2 \operatorname{arctg} \sqrt{\frac{\xi_{1}^{2} \xi_{2}^{\prime 2}-\left(\xi_{1} \cdot \xi_{2}^{\prime}\right)^{2}}{\left(\xi_{1} \cdot \xi_{2}^{\prime}\right)^{2}}} \quad \text { if } \quad \xi_{1}^{\prime}=0, \xi_{2}^{\prime 2}<0, \xi_{1} \cdot \xi_{2}^{\prime}>0 \\
& 2 \operatorname{arctg} \sqrt{\frac{\xi_{1}^{\prime 2} \xi_{2}^{2}-\left(\xi_{1}^{\prime} \cdot \xi_{2}\right)^{2}}{\left(\xi_{1}^{\prime} \cdot \xi_{2}\right)^{2}}} \quad \text { if } \quad \xi_{1}^{\prime 2}<0, \xi_{2}^{\prime}=0 \xi_{1}^{\prime} \cdot \xi_{2}>0 \text {. }
\end{aligned}
$$

In particular, $w_{2}^{T}\left(\lambda ; \xi_{i}, \xi_{i}^{\prime}\right)$ can be analytically continued in $\lambda$ into a half-plane for all $\left(\xi_{1}, \xi_{2}, \xi_{1}^{\prime}, \xi_{2}^{\prime}\right) \in S_{2}$ :

$S_{2}=\left\{\left(\xi_{1}, \xi_{2}, \xi_{1}^{\prime}, \xi_{2}^{\prime}\right) / \xi_{1}^{\prime}, \xi_{2}^{\prime} \in\right.$ a plane that contains a time-like vector,

$$
\begin{aligned}
& \left.\xi_{i}^{\prime} \cdot \xi_{j}^{\prime}<0 \quad i, j=1,2\right\} \\
& \cup\left\{\left(\xi_{1}, \xi_{2}, \xi_{1}^{\prime}, \xi_{2}^{\prime}\right) / \xi_{1}^{\prime}=0, \quad \xi_{2}^{\prime 2}<0, \quad \xi_{1}^{2}<0, \quad \xi_{1} \xi_{2}^{\prime}<0\right\} \\
& \cup\left\{\left(\xi_{1}, \xi_{2}, \xi_{1}^{\prime}, \xi_{2}^{\prime}\right) / \xi_{1}^{\prime 2}<0, \quad \xi_{2}^{\prime}=0, \quad \xi_{2}^{2}<0, \quad \xi_{1}^{\prime} \cdot \xi_{2}<0\right\} .
\end{aligned}
$$

This analyticity domain together with the temperedness implies according to theorem $5.1 .12[7]$ that $w_{2}^{T}\left(\lambda ; \xi_{i}, \xi_{i}^{\prime}\right)$ for $\left(\xi_{1}, \xi_{2}, \xi_{1}^{\prime}, \xi_{2}^{\prime}\right) \in S_{2}$ can decrease at most (linearly) exponentially, i.e.

$$
\lim _{\lambda \rightarrow+\infty} \frac{\log \left|w_{2}^{T}\left(\lambda ; \xi_{i}, \xi_{i}^{\prime}\right)\right|}{\lambda}=-M_{2}\left(\xi_{i}, \xi_{i}^{\prime}\right)>-\infty
$$

unless $w_{2}^{T}\left(\lambda ; \xi_{i}, \xi_{i}^{\prime}\right) \equiv 0$.

It is not difficult now to treat the general truncated $n$-point function by the same method. One considers

$$
W_{n}^{T}\left(x_{0}, \ldots, x_{n}\right)=\left\langle A\left(x_{0}\right) \ldots A\left(x_{n}\right)\right\rangle^{T}
$$

for $x_{i-1}-x_{i}=\xi_{i}+\lambda \xi_{i}^{\prime} i=1, \ldots, n$ with $x_{0}, \ldots, x_{n}$ totally space-like in the order $0,1, \ldots, n-1, n$ for sufficiently large positive $\lambda, \xi_{i}$ and $\xi_{i}^{\prime}$ fixed, not all $\xi_{i}^{\prime}=0$.

$w_{n}^{T}\left(\lambda ; \xi_{i}, \xi_{i}^{\prime}\right)=W_{n}^{T}\left(x_{0}, \ldots, x_{n}\right)$ is real-analytic for sufficiently large positive $\lambda$ and can be analytically continued in $\lambda$ into a half-plane for all

$$
\left(\xi_{i_{i=1, \ldots, n}}, \xi_{i_{i=1, \ldots, n}}^{\prime}\right) \in S_{n}
$$


$S_{n}=S_{n}^{(1)} \cup S_{n}^{(2)} \cup S_{n}^{(3)}$

$S_{n}^{(1)}=\left\{\left(\xi_{i_{i=1, \ldots, n}}, \xi_{i_{i=1, \ldots, n}}^{\prime}\right) / \xi_{i}^{\prime} \in\right.$ a plane that contains a time-like vector, $\xi_{i}^{\prime} \cdot \xi_{j}^{\prime}<0$ for all $\left.i, j \in(1, \ldots, n)\right\}$

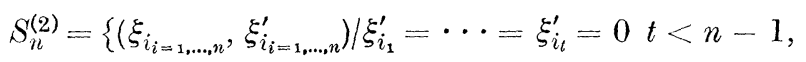

$\xi_{i \notin\left(i_{1}, \ldots, i_{t}\right)}^{\prime} \in$ a plane that contains a time-like vector, not all of the $\xi_{i_{i \notin\left(i_{1}, \ldots, i_{t}\right)}^{\prime}}^{\prime}$ on a space-like line, $\xi_{i}^{\prime} \cdot \xi_{j}^{\prime}<0$ for all $i, j \notin\left(i_{1}, \ldots, i_{t}\right)$, $\xi_{i} \cdot \xi_{j}^{\prime}<0$ for all $i \in\left(i_{1}, \ldots, i_{t}\right), j \notin\left(i_{1}, \ldots, i_{t}\right),\left(\xi_{j}^{\prime}\left(\xi_{i}, \xi_{l}^{\prime}\right)\right.$ $\left.-\xi_{l}^{\prime}\left(\xi_{i}, \xi_{j}^{\prime}\right)\right)^{2}>0$ for all $i \in\left(i_{1}, \ldots, i_{t}\right)$ and for at least two linearly independent $\xi_{j}^{\prime}, \xi_{l}^{\prime} j, l \notin\left(i_{1}, \ldots, i_{t}\right), \xi_{i}^{2}<0$ for all $\left.i \in\left(i_{1}, \ldots, i_{t}\right)\right\}$, $S_{n}^{(3)}=\left\{\left(\xi_{i_{i=1, \ldots, n}}, \xi_{i_{i=1, \ldots, n}}^{\prime}\right) / \xi_{i_{1}}^{\prime}=\cdots=\xi_{i_{t}}^{\prime}=0 \quad t<n\right.$, with $\xi_{i_{i \notin\left(i_{1}, \ldots, i_{l}\right)}^{\prime}}$ on a space-like line, $\xi_{i}^{\prime} \cdot \xi_{j}^{\prime}<0$ for all $i, j \notin\left(i_{1}, \ldots, i_{t}\right), \xi_{i} \cdot \xi_{j}^{\prime}<0$ for all $i \in\left(i_{1}, \ldots, i_{t}\right), j \notin\left(i_{1}, \ldots, i_{t}\right)$, there exists a time-like vector $\eta, \eta^{0}>0$ such that $\left(\eta\left(\xi_{i}, \xi_{j}^{\prime}\right)\right.$ $\left.-\xi_{j}^{\prime}\left(\xi_{i}, \eta\right)\right)^{2}+\xi_{i}^{2}\left(\eta, \xi_{j}^{\prime}\right)^{2}>0$ for all $\left.i \in\left(i_{1}, \ldots, i_{t}\right), j \notin\left(i_{1}, \ldots, i_{t}\right)\right\}$.

Again we may invoke the theorem 5.1.12 of [7] and conclude that for all $\left(\xi_{i_{i=1, \ldots, n}}, \xi_{i_{i=1, \ldots, n}}^{\prime}\right) \in S_{n} w_{n}^{T}\left(\lambda ; \xi_{i}, \xi_{i}^{\prime}\right)$ can decrease at most (linearly) exponentially

$$
\limsup _{\lambda \rightarrow+\infty} \frac{\log \left|w_{n}^{T}\left(\lambda ; \xi_{i}, \xi_{i}^{\prime}\right)\right|}{\lambda}=-M_{n}\left(\xi_{i}, \xi_{i}^{\prime}\right)>-\infty
$$

unless $w_{n}^{T}\left(\lambda ; \xi_{i}, \xi_{i}^{\prime}\right) \equiv 0$.

More detailed information about the decrease of $w_{n}^{T}\left(\lambda ; \xi_{i}, \xi_{i}^{\prime}\right)$ for $\left(\xi_{i}, \xi_{i}^{\prime}\right) \in S_{n}$ is given by the following theorem 10.4.1 of [7]: For each positive $\varepsilon$ and $\delta$ there is a sequence $\left\{\lambda_{n}\right\}, \lambda_{n} \rightarrow \infty$ and a positive $\eta$ such that the subset of $\left(\lambda_{n}, \lambda_{n}+\delta \lambda_{n}\right)$ in which

$$
\lambda^{-1} \log \left|w_{n}^{T}\left(\lambda ; \xi_{i}, \xi_{i}^{\prime}\right)\right| \geqq M_{n}\left(\xi_{i}, \xi_{i}^{\prime}\right)-\varepsilon
$$

has measure $\geqq \eta \cdot \lambda_{n}$.

\section{References}

1. Dell'Antonio, G. F., and P. Gullimanelli: Nuovo Cimento 12, 38 (1959).

2. Araki, H.: Ann. Phys. 11, 260 (1960).

3. Jost, R., and K. HePp: Helv. Phys. Acta 35, 34 (1962).

4. Ruelle, D.: Helv. Phys. Acta 35, 147 (1962).

5. Araki, H., K. Hepp, and D. Ruelle: Helv. Phys. Acta 35, 164 (1962).

6. Källén, G., and A. S. Wightman: Dan. Vid. Selsk. Mat. Fys. Skr. $1 n^{\circ} 6$ (1958).

7. BoAs, R. P.: Entire functions. New York: Academic Press Inc. 1954.

Dr. K. Pohlmeyer

II. Institut für Theoretische Physik der Universität 